\title{
Barium 2-Ketolactobionate and the Corresponding Barium Bromide Double Salt ${ }^{12}$
}

\author{
By William W. Walton and Horace S. Isbell
}

\begin{abstract}
As a preliminary step in the development of methods for the preparation of 2-ketoaldobionic acids for use in the synthesis of glycosidic derivatives of ascorbic acid, the preparation of 2-ketolactobionic acid has been studied. By oxidation of lactose osone with bromine in the presence of barium carbonate, barium 2-ketolactobionate was obtained and separated in the crystalline state. In addition to the normal barium salt, a double salt containing barium bromide was found. This salt is unique in that it is the first and only crystalline barium bromide salt of a sugar acid reported in the literature. It crystallizes freely and is useful for the separation and identification of 2-ketolactobionic acid.
\end{abstract}

\section{Introduction}

In consideration of the relationship between the carbohydrates and vitamin $\mathrm{C}$ and the prevalence of glycosides in natural products, it seemed desirable to undertake the preparation and study of some glycosidic derivatives of ascorbic acid. The most natural course for the synthesis of the glycosidic derivatives of ascorbic acid is through the intermediate production of 2-ketoaldobionic acids. The esters of certain 2-keto acids undergo lactonization and enolization with the formation of ascorbic acids $[1,2,3] .^{3}$ Thus one might expect that methyl 2-ketomelibionate on treatment with sodium methylate in methanol would give the following reaction:<smiles>CCOCCOC(=O)C(=O)C(O)C(O)C(O)COCCO</smiles>

Methyl 2-ketomelibionate<smiles>CCCCCOCC(O)C(O)C(O)C(O)C(=O)CO</smiles>

6-galactosylisoascorbic acid

The facility with which esters of 2-ketoaldonic acids yield ascorbic acid derivatives has led to the development of methods for their production. The most generally used method consists of oxidation of ketoses with potassium permanganate. With this method, it is necessary to protect the hydroxyl groups other than those attached to carbons one and two, as for instance by preparing acetone derivatives. It has been found, however, that L-sorbose [4] and D-fructose [5] can be oxidized directly with nitric acid to 2-keto-L-gluconic acid and 2keto-D-gluconic acid, respectively. In some cases the aldonic acids or their esters can be oxidized, and the keto acid or its ester can be obtained directly. This has been accomplished by several methods, notably by anodic oxidation of a salt of the aldonic acid [6] and by oxidation of the aldonic acid, its lactone, or ester with a chlorate in the presence of vanadium pentoxide [7]. The latter method requires the presence of an acid and is not suitable for the preparation of keto acids containing easily hydrolyzable glycosidic groups.

A method that does not require the presence of an acid consists of the production of the sugar osone followed by oxidation with bromine in sub-

\footnotetext{
${ }^{1}$ Abstracted from a thesis submitted by William W. Walton in June 1947 to the faculty of the Graduate School of the University of Maryland, represented by Nathan L. Drake, in partial fulfillment of the requirements for the degree of Doctor of Philosophy.

${ }^{2}$ Presented before the Division of Sugar Chemistry and Technology of the American Chemical Society at New York, Sept. 1947.

${ }^{3}$ Figures in brackets indicate the literature references at the end of this paper.
} 
stantially neutral solution. The procedure is suitable for use with osones derived from both monosaccharides and disaccharides, and it has been applied to a number of osones including maltosone [8]. The product from maltosone was reported to be amorphous and was undoubtedly an impure salt of 2-ketomaltobionic acid. As mentioned before, the enolization and lactonization of certain 2-ketoaldobionic acids would be expected to yield ascorbic acid derivatives of the desired type. 2-Ketomaltobionic acid and 2-ketolactobionic acid, however, contain substituents on the fourth carbon atom that prevent ring closure to that carbon atom. For this reason they cannot yield ascorbic acid derivatives. Nevertheless, on account of the ready availability of lactose it was selected for preliminary study of oxidation methods.

\section{Barium 2-Ketolactobionate and Its Barium Bromide Double Salt}

As an intermediate in the preparation of 2-ketolactobionic acid, it was necessary to prepare lactose osone. Ordinarily osones are obtained by reaction of phenylhydrazine with the sugar followed by treatment of the resulting phenylosazone with benzaldehyde [9] to remove the phenylhydrazine. In some cases osones have been obtained by direct oxidation of the sugar with copper acetate [10]; but this method is not applicable to disaccharides, because the reaction is slow and considerable hydrolysis takes place. Hence, the first method was adopted. The lactose osone was oxidized with bromine in the presence of barium carbonate, the resulting barium bromide was removed by treatment with silver sulphate, and barium 2-ketolactobionate was crystallized from the solution. Barium 2-ketolactobionate is the first crystalline metallic salt of a 2-ketoaldobionic acid to be prepared. It is quite soluble and does not crystallize easily. It was found, however, that barium 2-ketolactobionate forms a double salt with barium bromide, which crystallizes readily. The salt appears to be somewhat analogous to calcium lactobionate-calcium bromide [11]. Nevertheless, it is unique in that it is the first and only crystalline salt of a sugar acid to contain barium bromide. Photomicrographs of the normal salt and the double salt are shown in figure 1 .
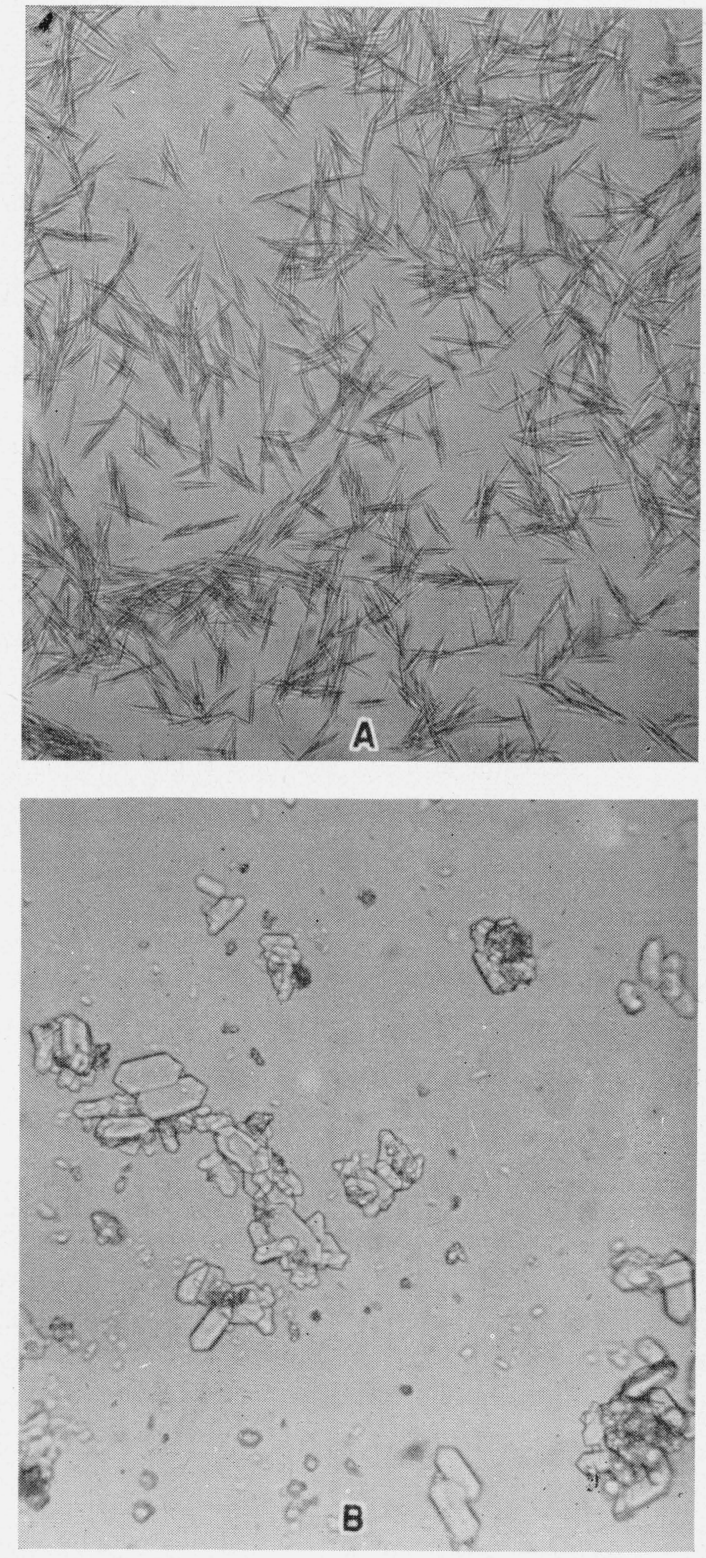

Figure 1. A, Barium 2-ketolactobionate dihydrate, $[\alpha]_{\mathrm{D}}^{20}=-37.9^{\circ}$, B, Barium 2-ketolactobionate-barium bromide tetrahydrate, $[\alpha]_{\mathrm{D}}^{20}=-28.2^{\circ} \cdot(\times 250)$.

The double salt is particularly suitable for the separation of 2 -ketolactobionic acid from the mixture obtained by the oxidation of lactose osone with bromine because it crystallizes freely from the impure mixture, and the barium bromide necessary for the formation of the salt is a byproduct of the reaction. The salt is characteristic of the 2-ketolactobionic acid structure and should be useful for identification purposes. 


\section{Structure of Barium 2-Ketolactobio- nate and its Barium Bromide Double Salt}

To establish that the normal barium 2-ketolactobionate and the barium bromide double salt are derivatives of the same 2-ketoaldobionic acid, the normal salt was prepared from the double salt by removal of the barium bromide with silver sulfate followed by crystallization. Furthermore, by the addition of a mole of barium bromide, the double salt was regenerated from the normal salt.

Since the synthesis began with lactose, (4- $\beta$-Dgalactosyl-D-glucose), in order to establish the structure of the new compound it was necessary (1) to prove that the biose linkage was still present and (2) to identify the products of hydrolysis as D-galactose and 2-keio-D-gluconic acid. Enzymatic hydrolysis of the substance with a sample of lactase ${ }^{4}$ established the existence of the biose linkage. Galactose was separated from the products of hydrolysis and identified by its melting point and optical rotation. Its presence in the hydrolytic mixture was also shown by the action of a galactose-fermenting yeast. The 2-ketogluconic acid was jdentified by separation of the characteristic phenylhydrazine salt of the phenylhydrazone.

This experimental work shows that the new compound is the barium salt of 4- $\beta$-D-galactosyl-2keto-D-gluconic acid (2-ketolactobionic acid). The freshly dissolved salt gave a specific rotation of $-36.4^{\circ}, 4$ minutes after dissolution, which changed to $-37.9^{\circ}$ in 45 minutes. The lack of a more extensive mutarotation is peculiar in light of the similarity of the substance to fructose and lactulose. 2-Ketolactobionic acid, like lactulose, has the fructose structure; it differs from lactulose (4- $\beta$-D-galactosyl-D-fructose) in having a carboxyl group in place of the terminal hydroxymethyl group. Fructose crystallizes in the pyranose form but establishes an equilibrium containing a substantial proportion of the furanose modification. Lactulose crystallizes in the furanose form, but its equilibrium state is very similar to that of fructose [12]. Since crystalline sugars having the fructose structure are thus known to exist in both furanose and pyranose forms, it is not possible to

\footnotetext{
4 The lactase was kindly supplied by the research laboratories of Rohm \& Haas, Philadelphia, Pa.
}

draw a conclusion concerning the ring structure of the crystalline barium salt by analogy to similar compounds.

It has been shown previously that an equilibrium state involving both pyranose and furanose modifications is particularly sensitive to changes in temperature, and that this equilibrium can be studied by measurements of optical rotation at various temperatures. A comparison of the equilibrium rotations of barium 2-ketolactobionate, lactulose, and levulose at two temperatures is given in table 1. In each case the optical rotation is appreciably altered by a change in temperature, and in the same direction. The like behavior of the compounds is evidence that they establish similar equilibrium states. Hence, barium 2ketolactobionate, like fructose and lactulose, establishes an equilibrium state containing substantial amounts of both pyranose and furanose modifications. The lack of a more extensive mutarotation for the salt must be explained by changes too rapid to be detected, or by the presence of more than one modification of the sugar entity in the barium salt at hand.

TABLE 1. Comparison of optical rotation

\begin{tabular}{|c|c|c|}
\hline \multirow{2}{*}{ Substance } & \multicolumn{2}{|c|}{ Equilibrium rotation } \\
\hline & $0^{\circ} \mathrm{C}$ & $20^{\circ} \mathrm{C}$ \\
\hline Barium 2-ketolactobionate & $a-42.3$ & -37.9 \\
\hline Lactulose & -24.8 & -23.0 \\
\hline Levulose ...... & -46.6 & -42.7 \\
\hline
\end{tabular}

a Temberature $0.6^{\circ} \mathrm{C}$.

\section{Experimental Details}

\section{Preparation of Lactose Phenylosazone}

One hundred and eighty grams of lactose was dissolved in 1 liter of water, and $164 \mathrm{ml}$ of phenylhydrazine and $328 \mathrm{ml}$ of a 50-percent aqueous solution of acetic acid were added. The mixture was heated in a water bath for 2 hours at $80^{\circ} \mathrm{C}$, cooled, and stored in a refrigerator overnight. The osazone was removed by filtration, washed with dilute acetic acid, water, and finally with ether. The product was recrystallized from 20percent aqueous alcohol. The yield was 38 percent. 


\section{Preparation of Lactose Osone}

Seventy grams of lactose phenylosazone was dissolved in 6 liters of hot water, $56 \mathrm{~g}$ of benzaldehyde was added, and the mixture was stirred vigorously while being heated on the steam bath in an atmosphere of nitrogen for 4 hours. The solution was cooled and filtered and the filtrate extracted several times with ether to remove excess benzaldehyde. The aqueous solution was then treated with a decolorizing carbon, filtered and concentrated under reduced pressure to a volume of $800 \mathrm{ml}$. The yield on several preparations was about 65 percent.

\section{Preparation of Barium 2-Ketolactobionate- Barium Bromide}

The aqueous solution of lactose osone obtained above, $800 \mathrm{ml}$ in volume, was saturated with carbon dioxide and cooled in an ice bath. Thirtyfive grams of barium carbonate and $12 \mathrm{ml}$ of bromine were added, and the mixture was stirred. After 5 minutes the flask was removed from the ice bath and the contents was stirred for $1 / 2$ hour at room temperature. Most of the excess bromine was removed with a rapid stream of carbon dioxide and the last trace by reaction with linseed oil. After separation from the oil, the mixture was filtered, treated with $10 \mathrm{~g}$ of a decolorizing carbon and refiltered. The colorless filtrate was concentrated under reduced pressure to a volume of approximately $50 \mathrm{ml}$ and transferred to a beaker with about 20 to $25 \mathrm{ml}$ of washings. In one preparation crystals separated at this point; in subsequent preparations the sirup was seeded and allowed to stand in a vacuum desiccator over sulfuric acid for a period of a day or cwo. The crystals were separated, washed with cold water, and air-dried. The yield in different preparations varied widely; the highest yield was $28.3 \mathrm{~g}$ and the lowest was $4 \mathrm{~g}$.

The material was recrystallized as follows: Twenty-five grams of the barium 2-ketolactobionate-barium bromide was dissolved in $475 \mathrm{ml}$ of water at room temperature, and the solution was filtered with the aid of a little decolorizing carbon. The filtrate was concentrated under reduced pressure to a volume of about $75 \mathrm{ml}$ and allowed to stand overnight at room temperature. The crystals that formed were collected on a funnel, washed with cold water and air-dried. Nineteen grams of pure barium 2-ketolactobionate-barium bromide was obtained. Analysis: Calculated for $\mathrm{Ba}\left(\mathrm{C}_{12} \mathrm{H}_{19} \mathrm{O}_{12}\right)_{2} \cdot \mathrm{BaBr}_{2} .4 \mathrm{H}_{2} \mathrm{O}: \mathrm{C}, 23.7 ; \mathrm{H}, 3.8 ; \mathrm{Ba}$, 22.6 ; Br, 13.1. Found: C, 23.9; H, 3.8; Ba, 22.6; $\mathrm{Br}$, 13.2. At equilibrium, the specific rotation $[\alpha]_{\mathrm{D}}^{20}=-28.2^{\circ}($ water $\mathrm{c}=2)$.

\section{Preparation of Barium 2-Ketolactobionate}

Barium 2-ketolactobionate was prepared by oxidation of lactose osone in a manner analogous to the preparation of the double salt. However, after treatment of the oxidized solution with carbon, the barium bromide was removed from the filtrate by treatment with silver sulfate and filtration. The solution was concentrated to about $50 \mathrm{ml}$ under reduced pressure, and $50 \mathrm{ml}$ of methanol was added. The solution was seeded with crystals of barium 2-ketolactobionate and allowed to crystallize for 2 days.

The material was recrystallized as follows: Seven grams of barium 2-ketolactobionate was dissolved in $20 \mathrm{ml}$ of water, and the solution was filtered with the aid of a little decolorizing carbon. About $25 \mathrm{ml}$ of methanol was slowly added to the filtrate and the solution was seeded and allowed to crystallize overnight. About 6 grams of recrystallized material was obtained. Analysis of the air-dried salt: Calculated for $\mathrm{Ba}\left(\mathrm{C}_{12} \mathrm{H}_{19} \mathrm{O}_{12}\right)_{2} .2 \mathrm{H}_{2} \mathrm{O}: \mathrm{C}, 32.6$; H, 4.8; Ba, 15.5. Found: C, 32.7; H, 4.8; Ba, 15.7. At equilibrium, the specific rotation $[\alpha]_{\mathrm{D}}^{20}=-37.9^{\circ}$ (water $\mathrm{c}=2$ ); $[\alpha]_{\mathrm{D}}^{0.6}=-42.3^{\circ}$.

\section{Structure of Barium 2-Ketolactobionate}

\section{(a) Enzymatic Hydrolysis}

A 1.105-g sample of barium 2-ketolactobionate was treated with an equivalent amount of $0.5 \mathrm{~N}$ sulfuric acid and the solution was stirred for 1 hour. Twenty-five hundredths gram of calcium carbonate was added, and the solution was stirred for 2 hours and filtered. The $\mathrm{pH}$ of the solution was adjusted to 6.7 with carbon dioxide. The optical rotation of the solution was $-8.55^{\circ} \mathrm{S}$ (4-dm tube). Three-tenths gram of lactase was then added, the solution was covered with toluene to prevent mold formation and stored at $30^{\circ} \mathrm{C}$ for 4 days. The optical rotation indicated that hydrolysis was 70 percent completed. An additional $0.3 \mathrm{~g}$ of lactase was added, and the solution was stored at $30^{\circ} \mathrm{C}$ for an additional 7 days The optical rotation at that time was $-1.32^{\circ} \mathrm{S}$ (4 dm tube), indicating complete hydrolysis. 
(b) Identification of Galactose

The hydrolyzed solution obtained in (a) was separated from the toluene and filtered. A small quantity of a galactose-fermenting yeast and $1 \mathrm{ml}$ of a nutrient medium were added, and the solution was stored at $37^{\circ} \mathrm{C}$ for 24 hours. The optical rotation indicated the destruction of 18 percent of the theoretical amount of galactose in 1 day. This solution was reserved for part (c).

In a separate experiment, $2 \mathrm{~g}$ of barium 2-ketolactobionate was hydrolyzed by dissolving it in $25 \mathrm{ml}$ of 7.5-percent sulfuric acid and heating the solution on the steam bath for 4 hours. About $2.5 \mathrm{~g}$ of calcium carbonate was added to the cool solution, and the mixture was allowed to stand overnight. It was then filtered and passed successively through a cation exchange resin and an anion exchange resin to remove calcium 2-ketogluconate. The filtrate was evaporated to a sirup, $10 \mathrm{ml}$ of methanol was added, and the solution was seeded with galactose and allowed to crystallize overnight. The crystals were separated, dried over calcium chloride, and weighed. A yield of 51 percent of the theoretical amount of galactose was obtained. The material was recrystallized from a water-methanol solution and the melting point found to be $165^{\circ} \mathrm{C}$. Mutarotation measurements were made, and the following values were found: initial rotation $[\alpha]_{\mathrm{D}}^{20}=140.5^{\circ}$; final rotation $[\alpha]_{\mathrm{D}}^{20}=80.2^{\circ}$; mutarotation constant 0.009 . The corresponding values reported for galactose are: melting point, $165^{\circ}$ to $167^{\circ} \mathrm{C}$; initial rotation, $[\alpha]_{\mathrm{D}}^{20}=150.7^{\circ}$; final rotation $[\alpha]_{\mathrm{D}}^{20}=80.2^{\circ}$; mutarotation constant 0.008 to 0.009 depending on the carbon dioxide content of the water. (c) Identification of 2-Ketogluconic Acid

The solution from (b) in which the galactose had been partially destroyed by fermentation was filtered and concentrated to a volume of $4 \mathrm{ml}$. Five drop of acetic acid and 5 drops of phenylhydrazine were added, and the solution was seeded with crystals of the phenylhydrazine salt of the phenylhydrazone of 2-ketogluconic acid. Crystals formed readily, and were separated by filtration, washed with ether, and dried. The melting point was $95^{\circ}$ to $97^{\circ} \mathrm{C}$ with decomposition. The melting point of the phenylhydrazine salt prepared from authentic 2-ketogluconic acid was $96^{\circ}$ to $98^{\circ} \mathrm{C}$ with decomposition. A mixed melting point of the two preparations was $95^{\circ}$ to $97^{\circ} \mathrm{C}$.

\section{References}

[1] T. Reichstein and A. Grüssner, Helv. chim. Acta, 17, 311 (1934).

[2] K. Maurer and B. Schiedt, Ber. deut. chem. Ges. 66, 1054 (1933).

[3] T. Reichstein, Helv. chim. Acta. 17, 996, 1003 (1934).

[4] British Patent 443,901 (Sept. 1, 1934).

[5] H. Ohle, H. Erlbach, and H. Carls, Ber. deut. chem. Ges. 67, 324, 555 (1934).

[6] R. Pasternack and P. Regna, U. S. Patent 2,222,155 (Nov. 19, 1940).

[7] R. Pasternack and P. Regna, U. S. Patent 2,207,991 (July 16, 1940)

[8] T. Kitasato, Biochem. Z. 207, 217 (1929).

[9] E. Fischer, Liebigs Ann. Chem. 288, 145 (1895); Ber. deut. chem. Ges. 35, 3142 (1902).

[10] I. Stone, U. S. Patent 2,206,374 (July 2, 1940).

[11] H. S. Isbell, J. Research NBS 17, 331 (1936) RP914.

[12] H. S. Isbel] and W. W. Pigman, J. Research NBS 20, 773 (1938) RP1104.

Washington, April 22, 1948. 